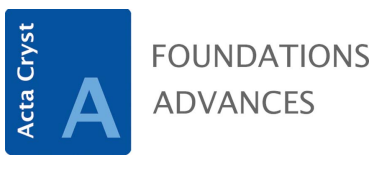

ISSN 2053-2733
Keywords: book review; dynamics of solid structures

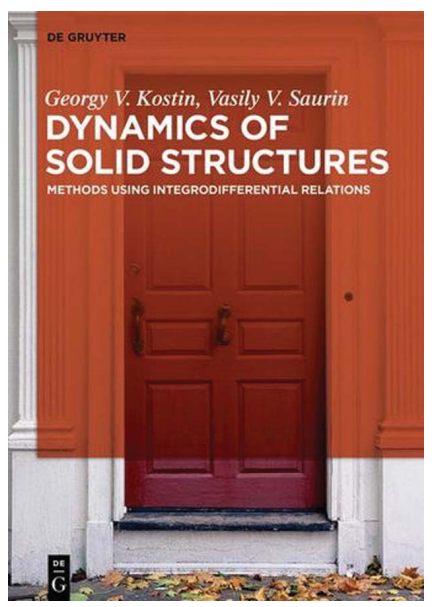

C 2019 International Union of Crystallography

\section{Dynamics of Solid Structures. Methods using Integrodifferential Relations. By Georgy Viktorovich Kostin and Vasily V. Saurin. De Gruyter, 2017. Hardcover, pp. xvii + 288. Price EUR 119.95, USD 137.99, GBP 109.00. ISBN 978-3-11-051623-4.}

\author{
Gregory Chirikjian*
}

Faculty of Engineering, Department of Mechanical Engineering, National University of Singapore, 9 Engineering Drive 1, Block EA \#07-08, 117575, Singapore. *Correspondence e-mail: mpegre@nus.edu.sg

This book builds on the authors' previous book (Kostin \& Saurin, 2012). Both books appear to be written for advanced graduate students in mechanics with an emphasis on the sub-discipline of integral/variational formulations of mechanics. The premise of these books is that such formulations are more robust with regard to discretization than classical partial-differential-equation formulations. Indeed, such methods (which are often called 'weak' formulations of mechanics) have been used for many decades in the context of finite element methods. This book is an attempt to add additional rigor to the very mature field of mechanics.

This new book is enigmatic in many ways. On the one hand, it builds on the previous book, repeating some material, but on the other hand it attempts to encompass much more, such as adding control theory as one of the topics. Though this is not reflected in the title of the book, there is a natural fit between variational calculus and optimal control. This connection has been made in many books focusing specifically on those topics.

Moreover, this book is not a textbook, since there are no problem sets and readers are assumed to know the underlying mechanics principles from which presented equations of motion have materialized. Also, the first four chapters are essentially devoid of figures such as free-body diagrams which would enlighten the reader as to the connection between the equations and the mechanics. These two features would scare away all but the most robust students. As a reference book or monograph it has value for a niche readership focused on these methods. The writing is clear and authoritative.

The beginning of the book is supposed to introduce the reader to the dynamics of flexible solids and apply variational methods as a means for solving important problems in mechanics. The middle part focuses on discretization such as Ritz and Galerkin methods. The final portion of the book shifts to control of mechanical systems such as moving a flexible beam. The bibliography is adequate, though in a field as old as variational mechanics, it would not be difficult to add two or three times as many references.

In summary, this book will be of interest to researchers in theoretical and computational solid mechanics. It is perhaps less appropriate for a crystallography audience.

\section{References}

Kostin, G. V. \& Saurin, V. V. (2012). Integrodifferential Relations in Linear Elasticity. Berlin: DeGruyter. 